\title{
w-Wiener Polynomials for Width Distance of Some Special Graphs
}

Ali A. Ali

Asma S. Aziz

College of Computer Science and Mathematics

University of Mosul, Iraq

Received on: 19/04/2007

Accepted on: 30/05/2007

\section{ABSTRACT}

Let $\mathrm{G}$ be a $\mathrm{k}_{0}$-connected graph , and let $\mathrm{d}_{\mathrm{w}}(\mathrm{u}, \mathrm{v}), 1 \leq \mathrm{w} \leq \mathrm{k}_{0}$, be the $\mathrm{w}$ width, distance between the two vertices $\mathrm{u}, \mathrm{v}$ in $\mathrm{G}$. The w-Wiener polynomial of the width distance of $\mathrm{G}$ is defined by:

$W_{w}(G ; x)=\sum_{u, v \in V(G)} x^{d_{w}(u, v)}$

$\mathrm{W}_{\mathrm{w}}(\mathrm{G} ; \mathrm{x})$ is obtained in this paper for some special graphs $\mathrm{G}$ such as a cycle, a wheel, a theta graph, a straight hexagonal chain , and Wagner graph .The diameter with respect to the width distance $-\mathrm{w}$, and the Wiener index for each such special graphs are also obtained in this paper.

Keywords: Wiener Polynomial, Width Distance, diameter, Wiener index.

$$
\begin{aligned}
& \text { متعددات حدود وينر - للمسافة العرضية لبعض البيانات الخاصة } \\
& \text { علي عزيز علي أسماء صلاح عزيز } \\
& \text { كلية علوم الحاسبات والرياضيات، جامعة الموصل }
\end{aligned}
$$

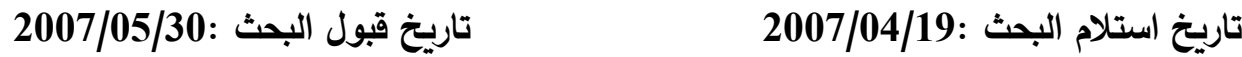

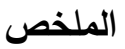

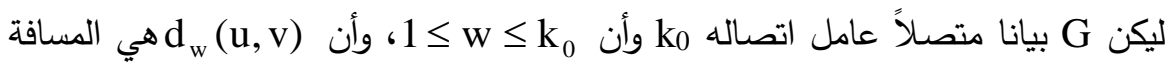

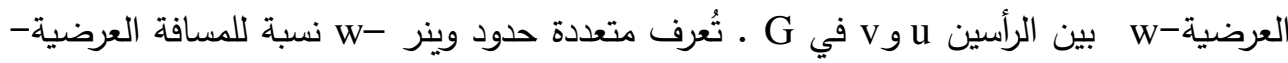

$$
\begin{aligned}
& \text { على أنها W } \\
& W_{w}(G ; x)=\sum_{u, v \in V(G)} x^{d_{w}(u, v)} \\
& \text { تضمن هذا البحث إيجاد Ww }
\end{aligned}
$$

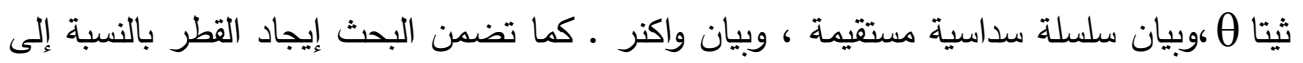

$$
\begin{aligned}
& \text { المسافة العرضية -w وإيجاد دليل وينر لكل من هذه البيانات الخاصة. }
\end{aligned}
$$

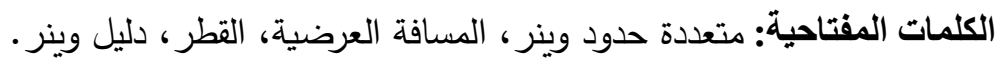


1.المقدمة Introduction

بسبب تطور فن تثييد شبكات الحاسوب ذوات الاتصال الداخلي المتوازي فقد أصبح

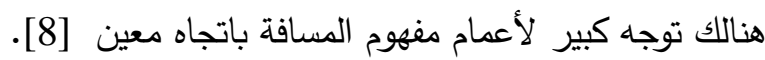

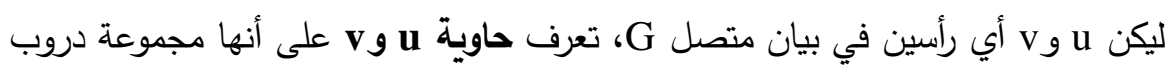

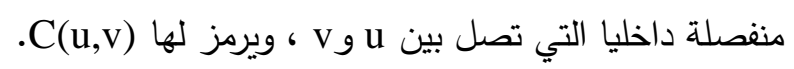

يعرف عرض(width) الحاوية C(u,v) على أنه عدد الدروب u-v في C(u,v) ويرمز له

w w (C(u,v))

$w(C(u, v))=|C(u, v)|$

كما يعرف طول الحاوية C(u,v) على أنه الطول لأطول درب في الحاوية ويرمز له

نعرف المسافة العرضية أو(المسافة-w (C(u,v))

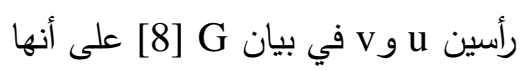

$d_{w}(u, v \mid G)=\min _{C(u, v)} \ell(C(u, v))$

حيث أن الأصغر يؤخذ على كل الحاويات C(u,v) ذات العرض w. وعندما لا يكون هناك التباس

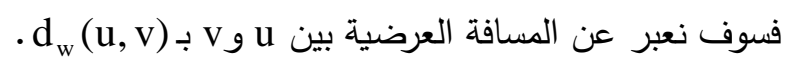

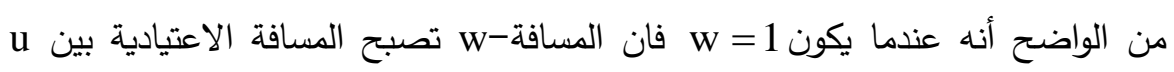

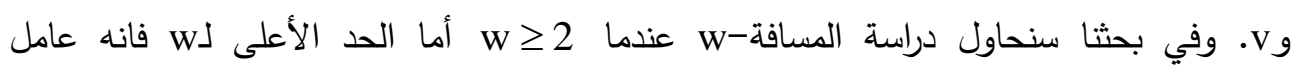

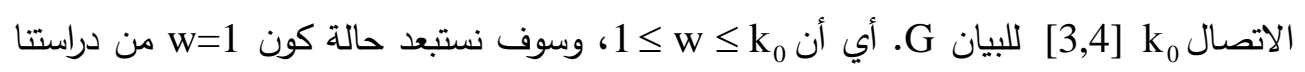

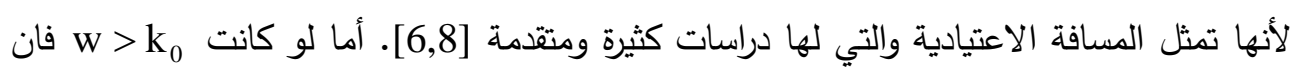

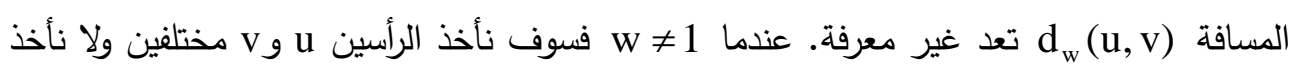
حالة كون u =v.

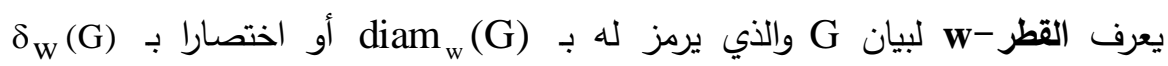

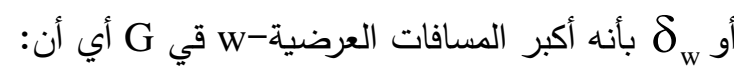

$\delta_{w}(G)=\max _{u, v \in V(G)} d_{w}(u, v \mid G)$

$$
\delta_{w}(G) \geq \delta(G) \text { واضح أن }
$$

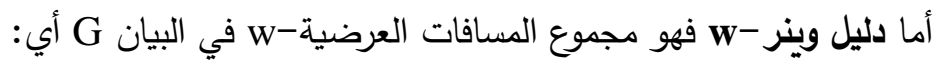

$W_{w}(G)=\sum_{u, v \in V} d_{w}(u, v \mid G)$ 
وأعماما لمتعددة حدود وينر نسبة لدالة المسافة الاعتيادية d [6] نعرف متعددة حدود

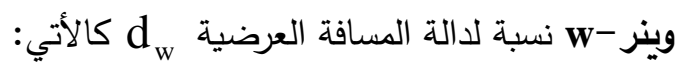

$W_{w}(G ; x)=\sum_{u, v \in V} x^{d_{w}(u, v)}$

فإذا كان $C_{w}$ يمثل عدد الأزواج غير المرتبة للرؤوس التي تساوي المسافة العرضية

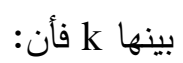
$W_{w}(G ; x)=\sum_{k \geq 2}^{\delta_{w}} C_{w}(G, k) x^{k}$

لأنه عندما يكون w فان المسافة-w لا تقل عن 2. من الواضح أن

$W_{w}(G)=\left.\frac{d}{d x} W_{w}(G ; x)\right|_{x=1}=\sum_{k \geq 2}^{\delta_{w}} k C_{w}(G, k)$

يعرف معدل المسافة العرضية-w بأنه حاصل قسمة دليل وينر w-w على عدد الأزواج

$$
\mu_{w}(G)=W_{w}(G) /\left(\begin{array}{l}
p \\
2
\end{array}\right)
$$$$
\text { غير المرتبة للرؤوس المختلفة وهو }
$$

ليكن v رأسا في بيان متصل G ولنغرض أن C مثل عدد رؤوس G (v, G,k التي

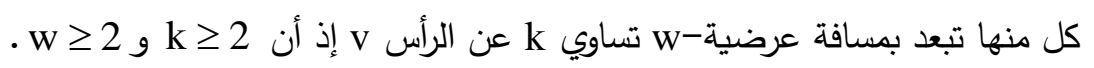

$\sum_{v \in V} C_{w}(v, G, k)=2 C_{w}(G, k)$

$$
2 \leq k \leq \delta_{w} \text { لكل }
$$

وتعرف متعددة حدود وينر - بالنسبة إلى الرأس V بـ

$W_{w}(v, G ; x)=\sum_{k \geq 2}^{\delta_{w}} C_{w}(v, G, k) x^{k}$

$$
\begin{aligned}
& \sum_{v \in V} W_{w}(v, G ; x)=2 W_{w}(G ; x) \\
& =2 \sum_{k \geq 2}^{\delta_{w}} C_{w}(G, k) x^{k}=2 W_{w}(G ; x) .
\end{aligned}
$$

من (1.5) و(1.8) و(1.9) نستنتج أن [1] 
تعريف: يقال لبيان متصل G أنه منتظم نسبة للمسـافة العرضية-w إذا كان لكل kل

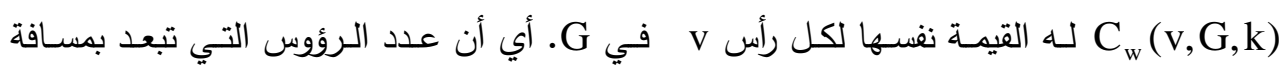

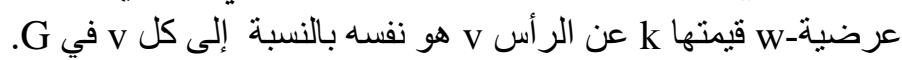

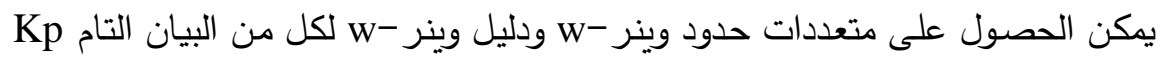
والثنائي التجزئة التام Kr,s كما هو مذكور أدناه:

$W_{w}\left(K_{p} ; x\right)=\frac{1}{2} p(p-1) x^{2}, \quad 2 \leq w \leq p-1$,

وعندئذ يكون

$W_{w}\left(K_{p}\right)=p(p-1)$

$W_{w}\left(K_{r, s} ; x\right)=\left[\left(\begin{array}{l}r \\ 2\end{array}\right)+\left(\begin{array}{l}s \\ 2\end{array}\right)\right] x^{2}+r s x^{3}, \quad 2 \leq w \leq \min \{r, s\}$,

$W_{w}\left(K_{r, s}\right)=r(r-1)+s(s-1)+3 r s$

$\mu_{w}\left(K_{r, s}\right)=W_{w}\left(K_{r, s}\right) /\left(\begin{array}{c}r+s \\ 2\end{array}\right)$

: $C_{p}$ الدارة

من الواضح أن عامل الاتصال للدارة C هو 2 وبذلك فان W=2. فإذا كان u و v أي رأسين مختلفين في C فان هناك دربين بين v و منفصيلين داخليا ومجموع طوليهما هو p.p. لذلك فان

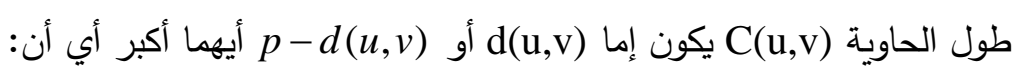
$\mathrm{d}_{2}(\mathrm{u}, \mathrm{v})=\max \{\mathrm{d}(\mathrm{u}, \mathrm{v}), \mathrm{p}-\mathrm{d}(\mathrm{u}, \mathrm{v})\}$

$1 \leq d(u, v) \leq\left\lfloor\frac{p}{2}\right\rfloor$

وبما أن

$d_{2}(u, v)=p-d(u, v)$

فان

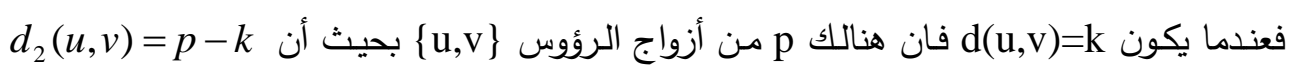
لقـيم \ $\cdot \frac{p}{2}$ \& $d_{2}(u, v)=\frac{p}{2}$ 
مما تقدم نستتتج متعددة حدود وينر ${ }^{2}{ }_{2}\left(C_{p} ; x\right)$ كما في المبرهنة الآتية:

مبرهنة 2.1 : لكل p

$W_{2}\left(C_{p} ; x\right)=\left\{\begin{array}{l}p\left(x^{p-1}+x^{p-2}+\ldots+x^{(p+1) / 2}\right), \quad \text { when } \mathrm{p} \text { is odd } \\ p\left(x^{p-1}+x^{p-2}+\ldots+x^{1+p / 2}+\frac{1}{2} x^{p / 2}\right), \text { when } \mathrm{p} \text { is even }\end{array}\right.$

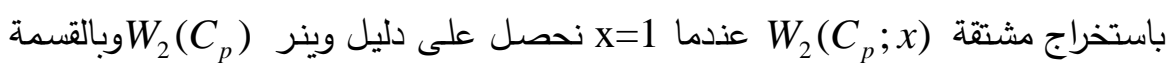

على $\left(\begin{array}{l}p \\ 2\end{array}\right)$

نتيجة 2.2 : لكل

$W_{2}\left(C_{p}\right)= \begin{cases}p(p-1)(3 p-1) / 8 ; & \text { when } \mathrm{p} \text { is odd } \\ \mathrm{p}^{2}(3 p-4) / 8 ; & \text { when } \mathrm{p} \text { is even }\end{cases}$

$\mu_{2}\left(C_{p}\right)=\left\{\begin{array}{lr}(3 p-1) / 4 ; & \text { when } \mathrm{p} \text { is odd } \\ \mathrm{p}(3 p-4) / 4(p-1) ; & \text { when } \mathrm{p} \text { is even }\end{array}\right.$

: W 3

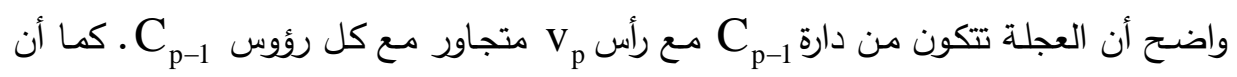

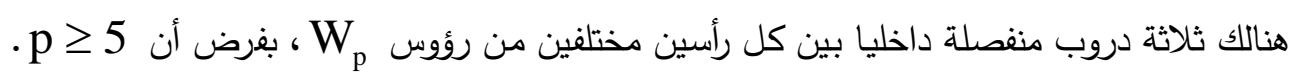

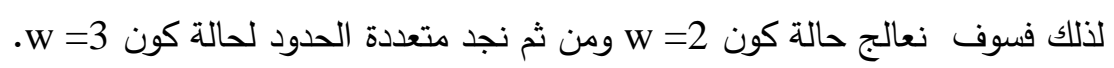

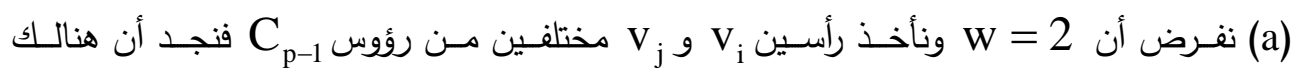

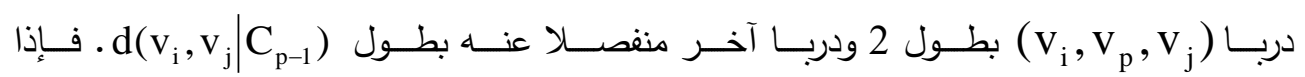
كان $v_{i} v_{j} \notin E\left(W_{p}\right)$

$\mathrm{d}_{2}\left(\mathrm{v}_{\mathrm{i}}, \mathrm{v}_{\mathrm{j}}\right)=\mathrm{d}\left(\mathrm{v}_{\mathrm{i}}, \mathrm{v}_{\mathrm{j}} \mid \mathrm{C}_{\mathrm{p}-1}\right)$

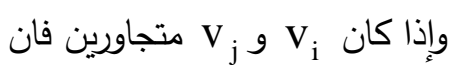

$\mathrm{d}_{2}\left(\mathrm{v}_{\mathrm{i}}, \mathrm{v}_{\mathrm{j}}\right)=2$

$\mathrm{d}_{2}\left(\mathrm{v}_{\mathrm{i}}, \mathrm{v}_{\mathrm{p}}\right)=2$

فضلا عن ذلك، فان 
لكل رأس V من رؤوس وعليه مما تقدم نستتتج أن: (3.4) (3ن)

$\mathrm{W}_{2}\left(\mathrm{~W}_{\mathrm{p}} ; \mathrm{x}\right)=2(\mathrm{p}-1) \mathrm{x}^{2}+\mathrm{W}\left(\mathrm{C}_{\mathrm{p}-1} ; \mathrm{x}\right)-(\mathrm{p}-1)(\mathrm{x}+1)$

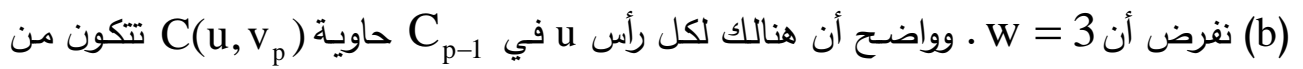

$\mathrm{d}_{3}\left(\mathrm{u}, \mathrm{v}_{\mathrm{p}}\right)=2, \quad \mathrm{u} \in \mathrm{V}\left(\mathrm{C}_{\mathrm{p}-1}\right)$

دربين بطول 2 مع درب بطول واحد وعليه فان

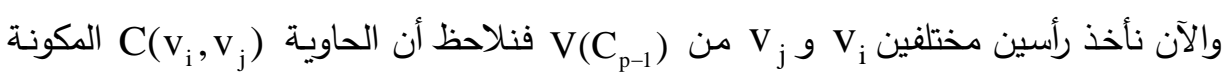
من ثلاثة دروب هي v مع دربي vi

$\mathrm{d}_{3}\left(\mathrm{v}_{\mathrm{i}}, \mathrm{v}_{\mathrm{j}}\right)=\mathrm{d}_{2}\left(\mathrm{v}_{\mathrm{i}}, \mathrm{v}_{\mathrm{j}} \mid \mathrm{C}_{\mathrm{p}-1}\right)$

وكذلك نستنتج من (3.5) و(3.6) أن:

$\mathrm{W}_{3}\left(\mathrm{~W}_{\mathrm{p}} ; \mathrm{x}\right)=(\mathrm{p}-1) \mathrm{x}^{2}+\mathrm{W}_{2}\left(\mathrm{C}_{\mathrm{p}-1} ; \mathrm{x}\right)$

وأخيرا باستخدام [6] ومبرهنة 1.2 نحصل على المبرهنة الآتية:

\section{مبرهنة3.1:}

لأجل p

(a)

$\mathrm{W}_{2}\left(\mathrm{~W}_{\mathrm{p}} ; \mathrm{x}\right)= \begin{cases}(\mathrm{p}-1)\left(3 \mathrm{x}^{2}+\mathrm{x}^{3}+\ldots+\mathrm{x}^{(\mathrm{p}-2) / 2}\right) ; & \text { when } \mathrm{p} \text { is even } \\ (\mathrm{p}-1)\left(3 \mathrm{x}^{2}+\mathrm{x}^{3}+\ldots+\mathrm{x}^{(\mathrm{p}-3) / 2}+\frac{1}{2} \mathrm{x}^{(\mathrm{p}-1) / 2}\right) ; & \text { when } \mathrm{p} \text { is odd }\end{cases}$

فان (b)

$\mathrm{W}_{3}\left(\mathrm{~W}_{\mathrm{p}} ; \mathrm{x}\right)=(\mathrm{p}-1) \mathrm{x}^{2}$

$+ \begin{cases}(\mathrm{p}-1)\left(\mathrm{x}^{\mathrm{p}-2}+\mathrm{x}^{\mathrm{p}-3}+\ldots+\mathrm{x}^{\mathrm{p} / 2}\right) ; & \text { when } \mathrm{p} \text { is even } \\ (\mathrm{p}-1)\left(\mathrm{x}^{\mathrm{p}-2}+\mathrm{x}^{\mathrm{p}-3}+\ldots+\mathrm{x}^{(\mathrm{p}+1) / 2}+\frac{1}{2} \mathrm{x}^{(\mathrm{p}-1) / 2}\right) ; \text { when } \mathrm{p} \text { is odd }\end{cases}$

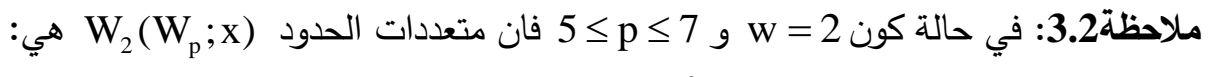

$$
\begin{aligned}
& \mathrm{W}_{2}\left(\mathrm{~W}_{5} ; \mathrm{x}\right)=10 \mathrm{x}^{2}, \\
& \mathrm{~W}_{2}\left(\mathrm{~W}_{6} ; \mathrm{x}\right)=15 \mathrm{x}^{2},
\end{aligned}
$$$$
\mathrm{W}_{2}\left(\mathrm{~W}_{7} ; \mathrm{x}\right)=18 \mathrm{x}^{2}+3 \mathrm{x}^{3} \text {, }
$$ 


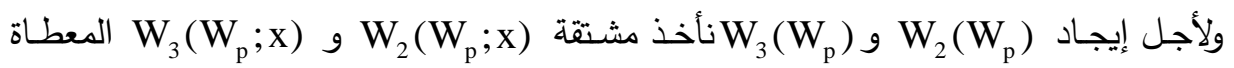

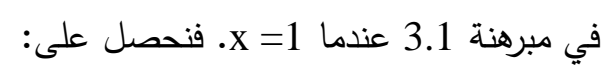

\section{نتيجة3.3:}

لأجل p a) $\mathrm{W}_{2}\left(\mathrm{~W}_{\mathrm{p}}\right)= \begin{cases}(\mathrm{p}-1)\left(\mathrm{p}^{2}-2 \mathrm{p}+24\right) / 8 ; & \text { when } \mathrm{p} \text { is even } \\ (\mathrm{p}-1)^{3} / 8+3(\mathrm{p}-1) ; & \text { when } \mathrm{p} \text { is odd }\end{cases}$ $\mathrm{W}_{3}\left(\mathrm{~W}_{\mathrm{p}}\right)= \begin{cases}(\mathrm{p}-1)\left(3 \mathrm{p}^{2}-10 \mathrm{p}+24\right) / 8 ; & \text { when } \mathrm{p} \text { is even } \\ (\mathrm{p}-1)\left(3 \mathrm{p}^{2}-10 \mathrm{p}+23\right) / 8 ; & \text { when } \mathrm{p} \text { is odd }\end{cases}$

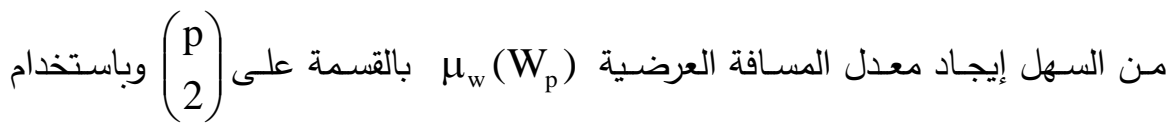
نتيجة 3.3وهو: $\mu_{2}\left(W_{p}\right)=\frac{1}{4 p} \begin{cases}\left(p^{2}-2 p+24\right) ; & \text { when } p \text { is even } \\ \left(p^{2}-2 p+25\right) ; & \text { when } p \text { is odd }\end{cases}$ $\mu_{3}\left(W_{p}\right)=\frac{1}{4 p} \begin{cases}\left(3 p^{2}-10 p+24\right) ; & \text { when } p \text { is even } \\ \left(3 p^{2}-10 p+23\right) ; & \text { when } p \text { is odd }\end{cases}$

Theta Graph: $(\theta)$ بيان ثيتا. تعريف:4.1: يعرف بيان

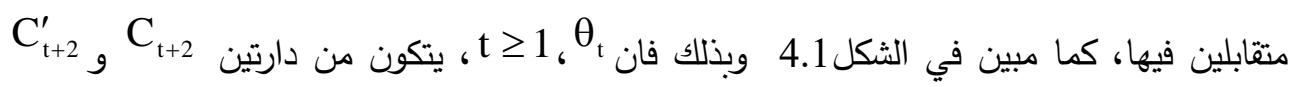

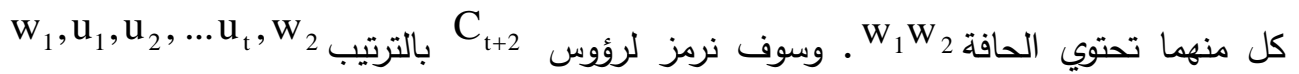

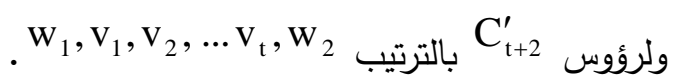




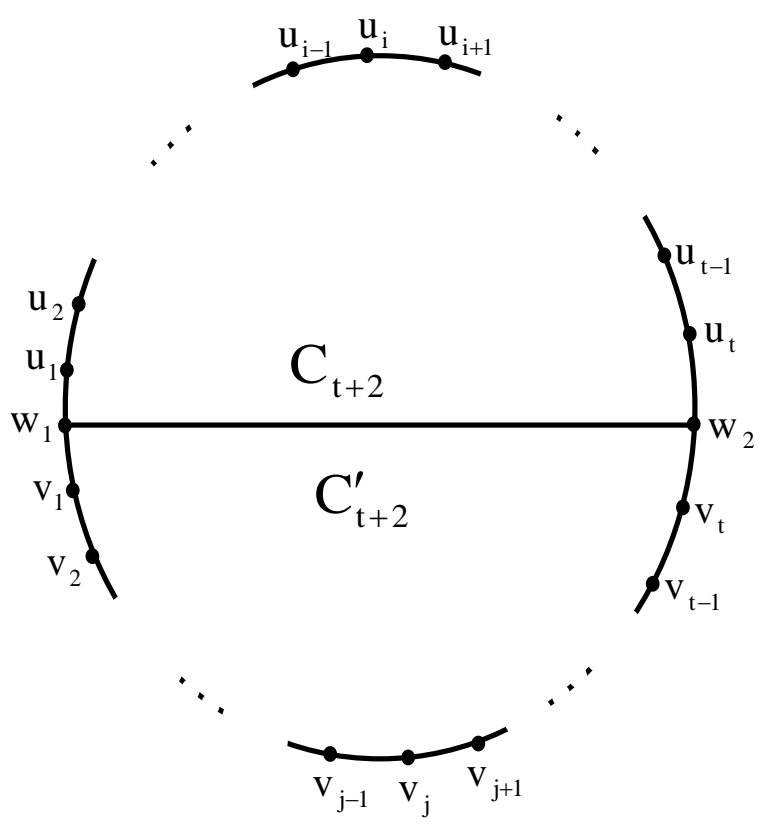

الثكل 4.1 بيان

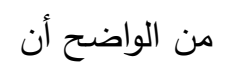

$\mathrm{p}\left(\theta_{\mathrm{t}}\right)=2(\mathrm{t}+1), \mathrm{q}\left(\theta_{\mathrm{t}}\right)=2 \mathrm{t}+3$

ومن ملاحظة الثكل1.4 يتبين لنا أن عامل الاتصال يساوي 2، وبذلك فان لدينا 2 ف فقط. ومن الواضح أن القطر للمسافة العرضية-2 هو لنافئن الأن $\operatorname{diam}_{2} \theta_{\mathrm{t}}=\mathrm{d}_{2}\left(\mathrm{u}_{\mathrm{t}}, \mathrm{v}_{\mathrm{t}}\right)=\mathrm{d}_{2}\left(\mathrm{u}_{1}, \mathrm{v}_{1}\right)=2 \mathrm{t}$

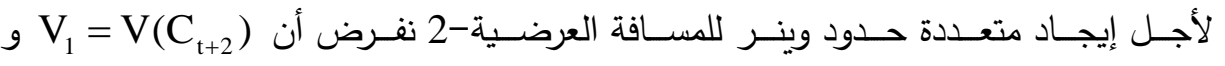

$$
\begin{aligned}
& \cdot \mathrm{V}_{2}=\mathrm{V}\left(\mathrm{C}_{\mathrm{t}+2}^{\prime}\right)
\end{aligned}
$$

إذا كان C(u, un

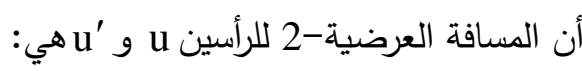
$\mathrm{d}_{2}\left(\mathrm{u}, \mathrm{u}^{\prime} \mid \theta_{\mathrm{t}}\right)=\mathrm{d}_{2}\left(\mathrm{u}, \mathrm{u}^{\prime} \mid \mathrm{C}_{\mathrm{t}+2}\right)$

وعليه فان متعددة الحدود لأزواج الرؤوس في ل هي: 
$\sum_{u, u^{\prime} \in V_{1}} x^{d_{2}\left(u, u^{\prime}\right)}=W_{2}\left(C_{t+2} ; x\right)$

وبالمثل فان متعددة الحدود لأزواج الرؤوس في V هي:

$\sum_{\mathrm{v}, \mathrm{v}^{\prime} \in \mathrm{V}_{2}} \mathrm{X}^{\mathrm{d}_{2}\left(\mathrm{v}, \mathrm{v}^{\prime}\right)}=\mathrm{W}_{2}\left(\mathrm{C}_{\mathrm{t}+2}^{\prime} ; \mathrm{x}\right)=\mathrm{W}_{2}\left(\mathrm{C}_{\mathrm{t}+2} ; \mathrm{x}\right)$

وبسبب تكرار الرأسين w و w $w_{2}$ $\mathrm{d}_{2}\left(\mathrm{w}_{1}, \mathrm{w}_{2} \mid \theta_{\mathrm{t}}\right)=\mathrm{t}+1$

C

ذات أقل طول (أي التي طولها يساوي المسافة العرضية-2 للزوج الآتيين:

$\mathrm{P}_{1}: \mathrm{u}_{\mathrm{i}}, \mathrm{u}_{\mathrm{i}-1}, \mathrm{u}_{\mathrm{i}-2}, \ldots, \mathrm{u}_{2}, \mathrm{u}_{1}, \mathrm{w}_{1}, \mathrm{v}_{1}, \mathrm{v}_{2}, \ldots, \mathrm{v}_{\mathrm{j}}$

$\mathrm{P}_{2}: \mathrm{u}_{\mathrm{i}}, \mathrm{u}_{\mathrm{i}+1}, \mathrm{u}_{\mathrm{i}+2}, \ldots, \mathrm{u}_{\mathrm{t}-1}, \mathrm{u}_{\mathrm{t}}, \mathrm{w}_{2}, \mathrm{v}_{\mathrm{t}}, \mathrm{v}_{\mathrm{t}-1}, \ldots, \mathrm{v}_{\mathrm{j}}$

$\ell\left(\mathrm{P}_{1}\right)=\mathrm{i}+\mathrm{j}$

من الواضح أن

$\ell\left(\mathrm{P}_{2}\right)=2 \mathrm{t}-\mathrm{i}-\mathrm{j}+2$

وأن

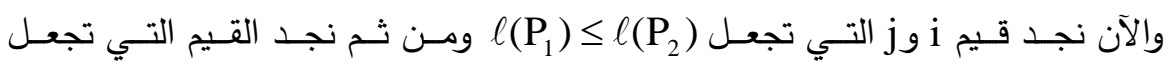
. $\ell\left(\mathrm{P}_{1}\right)>\ell\left(\mathrm{P}_{2}\right)$

$\mathrm{i}+\mathrm{j} \leq 2 \mathrm{t}-\mathrm{i}-\mathrm{j}+2$

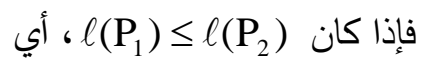

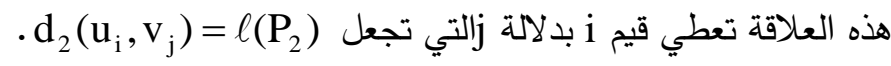

$2 \mathrm{i} \leq 2 \mathrm{t}-2 \mathrm{j}+2$

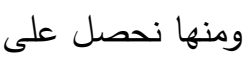

$\Rightarrow \mathrm{i} \leq \mathrm{t}-\mathrm{j}+1$

$\Rightarrow 1 \leq \mathrm{i} \leq \mathrm{t}-\mathrm{j}+1$

$$
\begin{aligned}
& \text { إن قيم i التي لا تحقق هذه المتباينة يكون فيها } \\
& \text { ومما تقدم وبالاعتماد على المتباينة (2.3.4) نحصل على: } \\
& \sum_{i=1}^{t} x^{d_{2}\left(u_{i}, v_{j}\right)}=\sum_{i=1}^{t-j+1} x^{2 t-i-j+2}+\sum_{i=t-j+2}^{t} x^{i+j}
\end{aligned}
$$




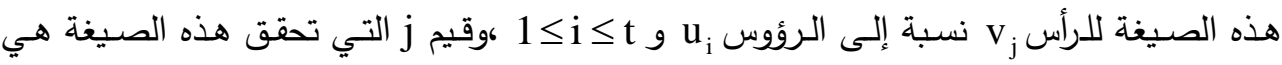

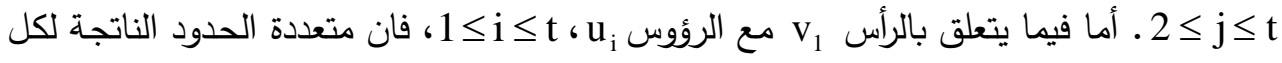

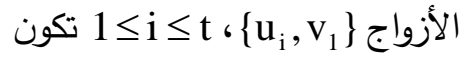
$\sum_{i=1}^{t} x^{d_{2}\left(v_{1}, u_{i}\right)}=\sum_{i=1}^{t} x^{2 t-i+1}$

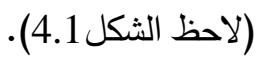

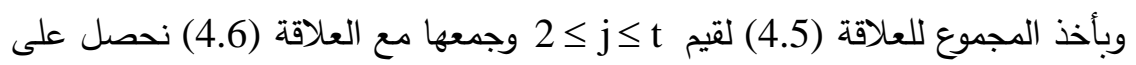

$\sum_{j=1}^{t} \sum_{i=1}^{t} x^{d_{2}\left(u_{i}, v_{j}\right)}=\sum_{j=2}^{t}\left(\sum_{i=1}^{t-j+1} x^{2 t-i-j+2}+\sum_{i=t-j+2}^{t} x^{i+j}\right)+\sum_{i=1}^{t} x^{2 t-i+1}$

وعند إجراء التبسيط الجبري على هذه العلاقة نحصل على الصيغة الآتية:

$\sum_{\mathrm{j}=1}^{\mathrm{t}} \sum_{\mathrm{i}=1}^{\mathrm{t}} \mathrm{x}^{\mathrm{d}_{2}\left(\mathrm{u}_{\mathrm{i}}, \mathrm{v}_{\mathrm{j}}\right)}=\left(\mathrm{x}^{\mathrm{t}+1}+\mathrm{x}^{\mathrm{t}+2}\right) \sum_{\mathrm{k}=0}^{\mathrm{t}-2}(\mathrm{t}-\mathrm{k}-1) \mathrm{x}^{\mathrm{k}}+\sum_{\mathrm{i}=1}^{\mathrm{t}} \mathrm{x}^{2 \mathrm{t}-\mathrm{i}+1}$

وبجمع العلاقتين (4.1) و(4.2) مع العلاقة (4.8) وطرح x+1 ، نحصل على متعددة حدود وينر

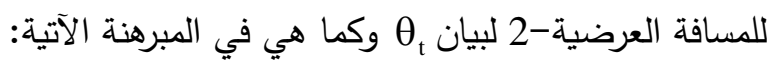

مبرهنة4.2: عندما تكون t 2 فان

$\mathrm{W}_{2}\left(\theta_{\mathrm{t}} ; \mathrm{x}\right)=2 \mathrm{~W}_{2}\left(\mathrm{C}_{\mathrm{t}+2} ; \mathrm{x}\right)-\mathrm{x}^{\mathrm{t}+1}+\left(\mathrm{x}^{\mathrm{t}+1}+\mathrm{x}^{\mathrm{t}+2}\right) \sum_{\mathrm{k}=0}^{\mathrm{t}-2}(\mathrm{t}-\mathrm{k}-1) \mathrm{x}^{\mathrm{k}}$ $+\sum_{i=1}^{t} x^{2 t-i+1}$

حيث أن

$\mathrm{W}_{2}\left(\mathrm{C}_{\mathrm{t}+2} ; \mathrm{x}\right)=\left\{\begin{array}{lr}(\mathrm{t}+2) \sum_{\mathrm{k}=(\mathrm{t}+3) / 2}^{\mathrm{t}+1} \mathrm{x}^{\mathrm{k}}, & \text { when } \mathrm{tis} \text { odd } \\ (\mathrm{t} / 2+1) \mathrm{x}^{\mathrm{t} / 2+1}+(\mathrm{t}+2) \sum_{\mathrm{k}=\mathrm{t} / 2+2}^{\mathrm{t}+1} \mathrm{x}^{\mathrm{k}}, & \text { when } \mathrm{t} \text { is even }\end{array}\right.$

\#

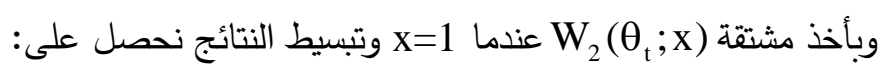

نتيجة4.3: دليل وينر للمسافة العرضية-2 لبيان t هو 
$\mathrm{W}_{2}\left(\theta_{\mathrm{t}}\right)= \begin{cases}(\mathrm{t} / 12)\left(25 \mathrm{t}^{2}+54 t+44\right)+1, & \text { when tis even } \\ (\mathrm{t} / 12)\left(25 \mathrm{t}^{2}+54 t+47\right)+3 / 2, & \text { when tis odd }\end{cases}$

5.بيان سلسلة سداسية مستقيمة: A graph of straight hexagonal chain

في اسـتخدام البيانـات لتمثيل جزئيـات مركبـات الكربوهيدرات نصـادف أنظمـة سداسـية

الحلقات متصلة مع بعضها البعض بأسلوب معين. وقد كانت هنالك دراسة لدليل وينر لهذه البيانات

[5,7]. وفي هذا البند ندرس نوعا معينا من هذه البيانات يسمى بيان سلسلة سداسية مستقيمة. تعريــ.15: يتكون البيان لسلسلة سداسية مستقيمة من عدد m من الحلقات السداسية كل منها يشترك مـع الحلقة التي تليها بحافة واحدة بحيث أن الخط الواصل بين مراكزها (على إعتبار أن

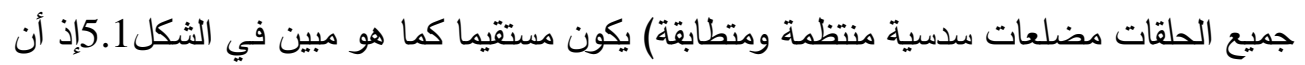

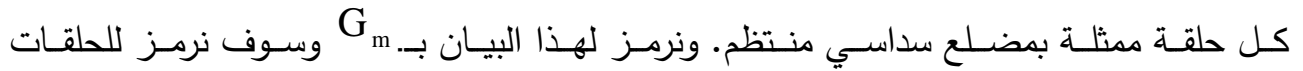
. $\mathrm{H}_{1}, \mathrm{H}_{2}, \ldots, \mathrm{H}_{\mathrm{m}}$.
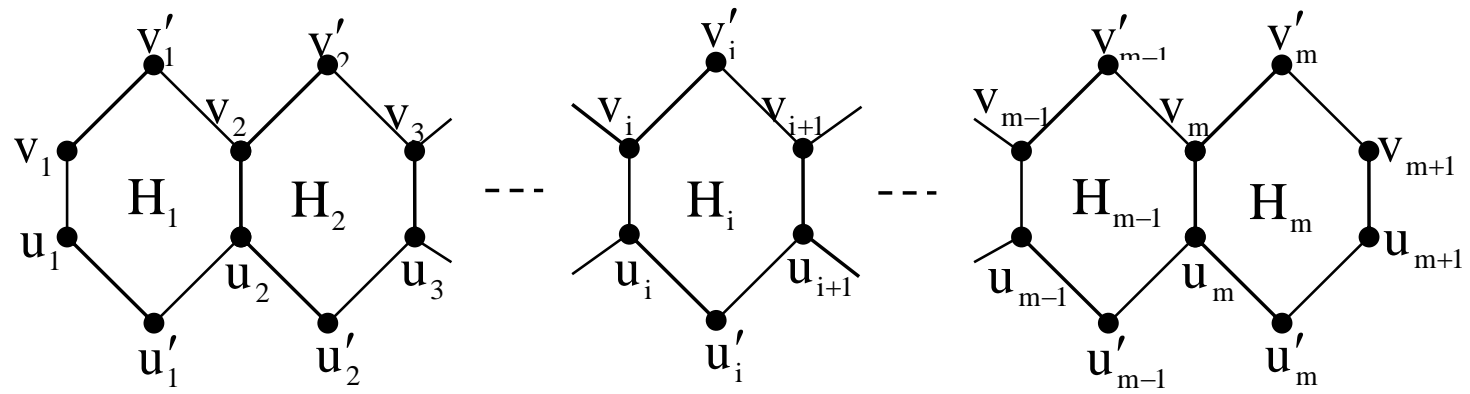

الثكل15.1

$$
\text { p }
$$

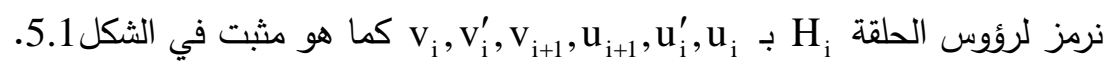
بملاحظة هذا البيان G نجد أن عامل الاتصال له هو 2، وعليه فسوف نجد المسافة

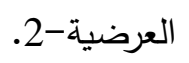

وفيما يتعلق بقطر المسـافة العرضية-2 (أي القطر -2) فانسه يسـاوي المسـافة العرضية بين

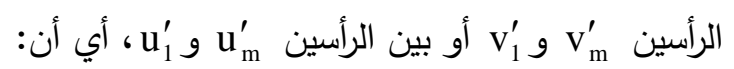
$\operatorname{diam}_{2} \mathrm{G}_{\mathrm{m}}=\max _{\mathrm{u}, \mathrm{v} \in \mathrm{V}\left(\mathrm{G}_{\mathrm{m}}\right)}\left\{\mathrm{d}_{2}(\mathrm{u}, \mathrm{v})\right\}=\mathrm{d}_{2}\left(\mathrm{v}_{1}^{\prime}, \mathrm{v}_{\mathrm{m}}^{\prime}\right)=\mathrm{d}_{2}\left(\mathrm{u}_{1}^{\prime}, \mathrm{u}_{\mathrm{m}}^{\prime}\right)$ 
$\operatorname{diam}_{2} \mathrm{G}_{\mathrm{m}}=2 \mathrm{~m}+4$

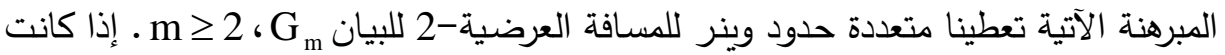

وان $\mathrm{G}_{1}=\mathrm{C}_{6}$ فان

$\mathrm{W}_{2}\left(\mathrm{C}_{6} ; \mathrm{x}\right)=6 \mathrm{x}^{5}+6 \mathrm{x}^{4}+3 \mathrm{x}^{3}$

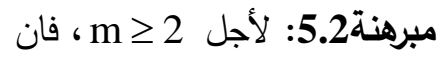

$\mathrm{W}_{2}\left(\mathrm{G}_{\mathrm{m}} ; \mathrm{x}\right)=3 \mathrm{~m}(1+2 \mathrm{x}) \mathrm{x}^{3}+(5 \mathrm{~m}+1) \mathrm{x}^{5}$

$+2\left(2+3 x+2 x^{2}+x^{3}\right) x^{3} \sum_{j=1}^{m-1}(m-j) x^{2 j}$

البرهان: لإثبات (5.3) نستخدم مبدأ الاستقراء الرياضي على m.

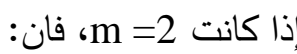

$\mathrm{W}_{2}\left(\mathrm{G}_{2} ; \mathrm{x}\right)=6 \mathrm{x}^{3}+12 \mathrm{x}^{4}+15 \mathrm{x}^{5}+6 \mathrm{x}^{6}+4 \mathrm{x}^{7}+2 \mathrm{x}^{8}$

ونفـرض أن متعددة حدود وينـر للمسـافة العرضـية-2

$$
\begin{aligned}
& \text {. } \mathrm{m} \geq 3 \text { لأجل } 3 \text { أل } \\
& \text { وبمقارنة G و G } \\
& \mathrm{W}_{2}\left(\mathrm{G}_{\mathrm{m}} ; \mathrm{x}\right)=\mathrm{W}_{2}\left(\mathrm{G}_{\mathrm{m}-1} ; \mathrm{x}\right)+\mathrm{W}_{2}\left(\mathrm{C}_{6} ; \mathrm{x}\right)+\mathrm{F}(\mathrm{x})-\mathrm{x}^{5} \\
& \mathrm{~F}(\mathrm{x})=\sum_{\mathrm{u}, \mathrm{v}} \mathrm{x}^{\mathrm{d}_{2}(\mathrm{u}, \mathrm{v})} \\
& \text { حيث أن }
\end{aligned}
$$

لكل

$\mathrm{v} \in \mathrm{V}\left(\mathrm{G}_{\mathrm{m}-1}\right)-\left\{\mathrm{v}_{\mathrm{m}}, \mathrm{u}_{\mathrm{m}}\right\}, \mathrm{u} \in \mathrm{A}=\left\{\mathrm{v}_{\mathrm{m}}^{\prime}, \mathrm{v}_{\mathrm{m}+1}, \mathrm{u}_{\mathrm{m}+1}, \mathrm{u}_{\mathrm{m}}^{\prime}\right\}$

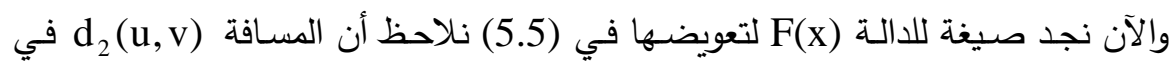

البيان G هي نفسها في الدارة

$\mathrm{u} \in \mathrm{A}, \mathrm{v} \in\left\{\mathrm{v}_{\mathrm{i}}, \mathrm{v}_{\mathrm{i}}^{\prime}, \mathrm{u}_{\mathrm{i}}, \mathrm{u}_{\mathrm{i}}^{\prime}\right\}$

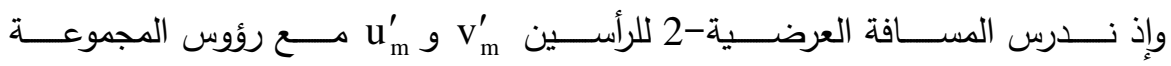

، عندما يكون A $A_{i}=\left\{v_{i}, v_{i}^{\prime}, u_{i}, u_{i}^{\prime}\right\}$

$\mathrm{d}_{2}\left(\mathrm{v}_{\mathrm{m}}^{\prime}, \mathrm{v}_{\mathrm{i}}\right)=\mathrm{d}_{2}\left(\mathrm{u}_{\mathrm{m}}^{\prime}, \mathrm{u}_{\mathrm{i}}\right)=5+2(\mathrm{~m}-\mathrm{i})$

$\mathrm{d}_{2}\left(\mathrm{v}_{\mathrm{m}}^{\prime}, \mathrm{v}_{\mathrm{i}}^{\prime}\right)=\mathrm{d}_{2}\left(\mathrm{u}_{\mathrm{m}}^{\prime}, \mathrm{u}_{\mathrm{i}}^{\prime}\right)=6+2(\mathrm{~m}-\mathrm{i})$

$\mathrm{d}_{2}\left(\mathrm{v}_{\mathrm{m}}^{\prime}, \mathrm{u}_{\mathrm{i}}\right)=\mathrm{d}_{2}\left(\mathrm{u}_{\mathrm{m}}^{\prime}, \mathrm{v}_{\mathrm{i}}\right)=4+2(\mathrm{~m}-\mathrm{i})$ 


$$
\begin{aligned}
& \mathrm{d}_{2}\left(\mathrm{v}_{\mathrm{m}}^{\prime}, \mathrm{u}_{\mathrm{i}}^{\prime}\right)=\mathrm{d}_{2}\left(\mathrm{u}_{\mathrm{m}}^{\prime}, \mathrm{v}_{\mathrm{i}}^{\prime}\right)=3+2(\mathrm{~m}-\mathrm{i})
\end{aligned}
$$

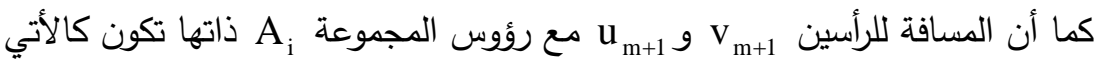

$$
\begin{aligned}
& \mathrm{d}_{2}\left(\mathrm{v}_{\mathrm{m}+1}, \mathrm{v}_{\mathrm{i}}\right)=\mathrm{d}_{2}\left(\mathrm{u}_{\mathrm{m}+1}, \mathrm{u}_{\mathrm{i}}\right)=4+2(\mathrm{~m}-\mathrm{i}) \\
& \mathrm{d}_{2}\left(\mathrm{v}_{\mathrm{m}+1}, \mathrm{v}_{\mathrm{i}}^{\prime}\right)=\mathrm{d}_{2}\left(\mathrm{u}_{\mathrm{m}+1}, \mathrm{u}_{\mathrm{i}}^{\prime}\right)=5+2(\mathrm{~m}-\mathrm{i}) \\
& \mathrm{d}_{2}\left(\mathrm{v}_{\mathrm{m}+1}, \mathrm{u}_{\mathrm{i}}\right)=\mathrm{d}_{2}\left(\mathrm{u}_{\mathrm{m}+1}, \mathrm{v}_{\mathrm{i}}\right)=3+2(\mathrm{~m}-\mathrm{i}) \\
& \mathrm{d}_{2}\left(\mathrm{v}_{\mathrm{m}+1}, \mathrm{u}_{\mathrm{i}}^{\prime}\right)=\mathrm{d}_{2}\left(\mathrm{u}_{\mathrm{m}+1}, \mathrm{v}_{\mathrm{i}}^{\prime}\right)=4+2(\mathrm{~m}-\mathrm{i})
\end{aligned}
$$

ومن هذه المسافات وبأخذ المجموع لكل i نستتتج صيغة F(x) وهي:$$
\mathrm{F}(\mathrm{x})=2 \sum_{\mathrm{i}=1}^{\mathrm{m}-1}\left(\mathrm{x}^{6+2(\mathrm{~m}-\mathrm{i})}+2 \mathrm{x}^{5+2(\mathrm{~m}-\mathrm{i})}+3 \mathrm{x}^{4+2(\mathrm{~m}-\mathrm{i})}+2 \mathrm{x}^{3+2(\mathrm{~m}-\mathrm{i})}\right)
$$

$$
\text { وبعد تعويضها في العلاقة (5.5) نحصل على: }
$$

$$
\begin{aligned}
\mathrm{W}_{2}\left(\mathrm{G}_{\mathrm{m}} ; \mathrm{x}\right) & =\mathrm{W}_{2}\left(\mathrm{G}_{\mathrm{m}-1} ; \mathrm{x}\right)+2 \sum_{\mathrm{i}=1}^{\mathrm{m}-1}\left(\mathrm{x}^{6+2(\mathrm{~m}-\mathrm{i})}+2 \mathrm{x}^{5+2(\mathrm{~m}-\mathrm{i})}+3 \mathrm{x}^{4+2(\mathrm{~m}-\mathrm{i})}\right. \\
& \left.+2 \mathrm{x}^{3+2(\mathrm{~m}-\mathrm{i})}\right)+\mathrm{W}_{2}\left(\mathrm{C}_{6} ; \mathrm{x}\right)-\mathrm{x}^{5}
\end{aligned}
$$

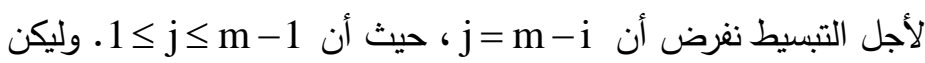

$z_{j}=x^{6+2 j}+2 x^{5+2 j}+3 x^{4+2 j}+2 x^{3+2 j}$

$$
\mathrm{W}_{2}\left(\mathrm{G}_{\mathrm{m}} ; \mathrm{x}\right)=\mathrm{W}_{2}\left(\mathrm{G}_{\mathrm{m}-1} ; \mathrm{x}\right)+\mathrm{W}_{2}\left(\mathrm{C}_{6} ; \mathrm{x}\right)-\mathrm{x}^{5}+2 \sum_{\mathrm{j}=1}^{\mathrm{m}-1} \mathrm{z}_{\mathrm{j}}
$$

$$
\mathrm{W}_{2}\left(\mathrm{G}_{\mathrm{m}-1} ; \mathrm{x}\right)=\mathrm{W}_{2}\left(\mathrm{G}_{\mathrm{m}-2} ; \mathrm{x}\right)+\mathrm{W}_{2}\left(\mathrm{C}_{6} ; \mathrm{x}\right)-\mathrm{x}^{5}+2 \sum_{\mathrm{j}=1}^{\mathrm{m}-2} \mathrm{z}_{\mathrm{j}}
$$

$$
\mathrm{W}_{2}\left(\mathrm{G}_{\mathrm{m}-2} ; \mathrm{x}\right)=\mathrm{W}_{2}\left(\mathrm{G}_{\mathrm{m}-3} ; \mathrm{x}\right)+\mathrm{W}_{2}\left(\mathrm{C}_{6} ; \mathrm{x}\right)-\mathrm{x}^{5}+2 \sum_{\mathrm{j}=1}^{\mathrm{m}-3} \mathrm{z}_{\mathrm{j}}
$$

$$
\begin{aligned}
& \mathrm{W}_{2}\left(\mathrm{G}_{3} ; \mathrm{x}\right)=\mathrm{W}_{2}\left(\mathrm{G}_{2} ; \mathrm{x}\right)+\mathrm{W}_{2}\left(\mathrm{C}_{6} ; \mathrm{x}\right)-\mathrm{x}^{5}+2 \sum_{\mathrm{j}=1}^{2} \mathrm{z}_{\mathrm{j}} \\
& \mathrm{W}_{2}\left(\mathrm{G}_{2} ; \mathrm{x}\right)=2 \mathrm{~W}_{2}\left(\mathrm{C}_{6} ; \mathrm{x}\right)-\mathrm{x}^{5}+2 \mathrm{z}_{1}
\end{aligned}
$$




$$
\begin{aligned}
& \mathrm{W}_{2}\left(\mathrm{G}_{\mathrm{m}} ; \mathrm{x}\right)=2\left(\sum_{\mathrm{j}=1}^{1} \mathrm{z}_{\mathrm{j}}+\sum_{\mathrm{j}=1}^{2} \mathrm{z}_{\mathrm{j}}+\sum_{\mathrm{j}=1}^{3} \mathrm{z}_{\mathrm{j}}+\ldots+\sum_{\mathrm{j}=1}^{\mathrm{m}-1} \mathrm{z} \mathrm{j}\right) \\
& +\mathrm{mW}_{2}\left(\mathrm{C}_{6} ; \mathrm{x}\right)-(\mathrm{m}-1) \mathrm{x}^{5} \\
& \therefore \mathrm{W}_{2}\left(\mathrm{G}_{\mathrm{m}} ; \mathrm{x}\right)=3 \mathrm{mx}^{3}\left(1+2 \mathrm{x}+2 \mathrm{x}^{2}\right)-(\mathrm{m}-1) \mathrm{x}^{5}+2 \sum_{\mathrm{j}=1}^{\mathrm{m}-1}(\mathrm{~m}-\mathrm{j}) \mathrm{z}_{\mathrm{j}} \\
& =3 m x^{3}(1+2 x)+(5 m+1) x^{5}+2 \sum_{j=1}^{m-1}(m-j) z_{j} \\
& \text { وبالتعويض عن Z المذكورة في (5.8) نحصل على (5.3) وبهذا يتم البرهان. } \\
& \text { نتيجة 5.3: دليل وينر للمسافة العرضية-2 للبيان }
\end{aligned}
$$$$
\mathrm{W}_{2}\left(\mathrm{G}_{\mathrm{m}}\right)=(16 / 3) \mathrm{m}\left(\mathrm{m}^{2}-4\right)+54 \mathrm{~m}^{2}+5
$$

بعد اثتقاق العلاقة (5.3) والتعويض عن x=1 نحصل على العلاقة (5.16) .

\section{6بون 6}

تعريف6.16: بيان واكنر [4]هو بيان مكون من دارة برتبة زوجية مع الحافات التي كل واحدة منها

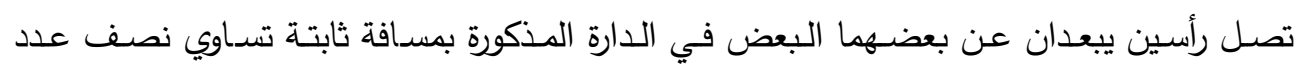
الرؤوس.

ولأجل توضيح هذا التعريف نفرض أن عدد الرؤوس يساوي t وأن t =2m. ونرمز لهذا البيان

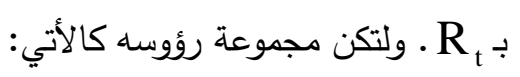
$\mathrm{V}\left(\mathrm{R}_{\mathrm{t}}\right)=\left\{\mathrm{v}_{1}, \mathrm{v}_{2}, \ldots, \mathrm{v}_{2 \mathrm{~m}}\right\}$

$\mathrm{E}\left(\mathrm{R}_{\mathrm{t}}\right)=\mathrm{E}\left(\mathrm{C}_{2 \mathrm{~m}}\right) \cup\left\{\mathrm{v}_{\mathrm{i}} \mathrm{v}_{\mathrm{m}+\mathrm{i}} \mid \mathrm{i}=1,2, \ldots, \mathrm{m}\right\}$

عندها تكون مجموعة حافاته

$$
\text { كما موضح في الثكل } 6.1 .
$$

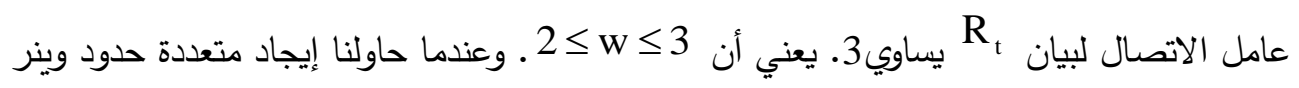

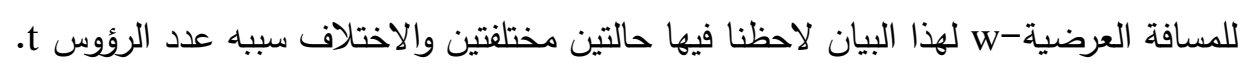

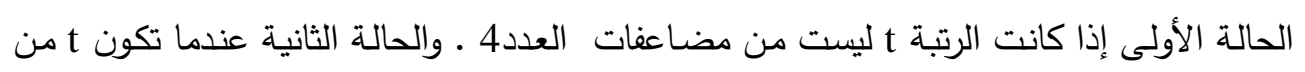
مضاعفات العدد4 . 
وأخيرا لا بد أن نذكر أن Rو هو بيان منتظم بالنسبة إلى المسافة العرضية. لهذا سنحاول

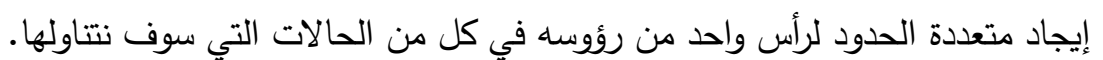

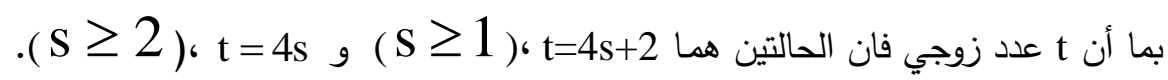
عبارة:6.2 إذا كانت t=4s+2 $\mathrm{W}_{2}\left(\mathrm{R}_{\mathrm{t}} ; \mathrm{x}\right)=(2 \mathrm{~s}+1) \mathrm{x}^{3}+\mathrm{tx} \sum_{\mathrm{i}=2}^{\mathrm{s}+1} \mathrm{x}^{\mathrm{i}}+\mathrm{t} \mathrm{x}^{2} \sum_{\mathrm{i}=s+2}^{2 s+1} \mathrm{x}^{2 s+1-\mathrm{i}}$

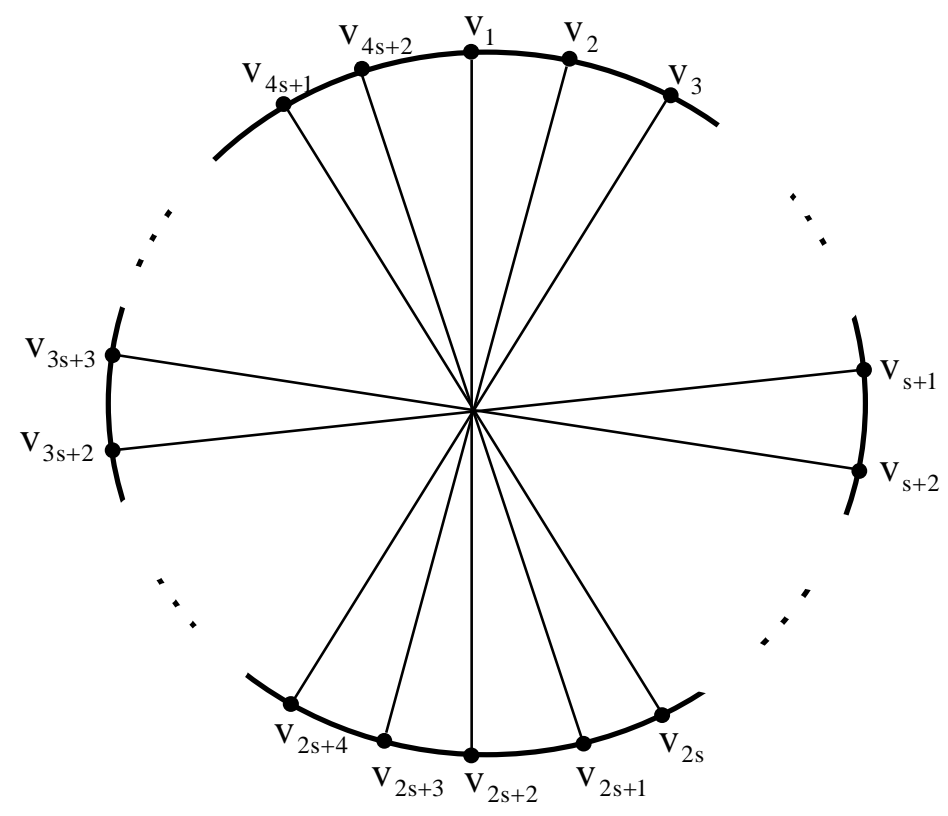

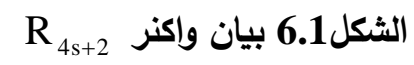

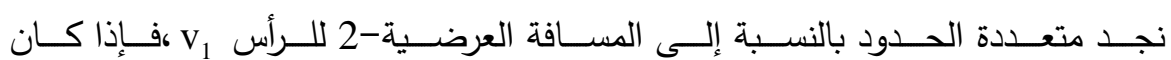

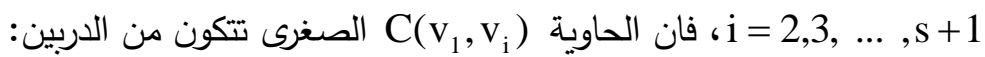
$\mathrm{P}_{1}: \mathrm{v}_{1}, \mathrm{v}_{2}, \mathrm{v}_{3}, \ldots, \mathrm{v}_{\mathrm{i}}$ $\mathrm{P}_{2}: \mathrm{v}_{1}, \mathrm{v}_{2 \mathrm{~s}+2}, \mathrm{v}_{2 \mathrm{~s}+3}, \ldots, \mathrm{v}_{2 \mathrm{~s}+\mathrm{i}+1}, \mathrm{v}_{\mathrm{i}}$

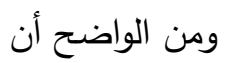

$$
\ell\left(\mathrm{P}_{1}\right)=\mathrm{i}-1, \ell\left(\mathrm{P}_{2}\right)=\mathrm{i}+1
$$


$\mathrm{d}_{2}\left(\mathrm{v}_{1}, \mathrm{v}_{\mathrm{i}}\right)=\max \left\{\ell\left(\mathrm{P}_{1}\right), \ell\left(\mathrm{P}_{2}\right)\right\}=\mathrm{i}+1$

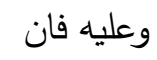

حيث

وإذا كانت

$$
\begin{aligned}
& \mathrm{P}_{1}: \mathrm{v}_{1}, \mathrm{v}_{2 \mathrm{~s}+2}, \mathrm{v}_{2 \mathrm{~s}+1}, \ldots, \mathrm{v}_{\mathrm{i}+1}, \mathrm{v}_{\mathrm{i}} \\
& \mathrm{P}_{2}: \mathrm{v}_{1}, \mathrm{v}_{4 s+2}, \mathrm{v}_{4 s+1}, \mathrm{v}_{4 \mathrm{~s}}, \ldots, \mathrm{v}_{2 s+1+\mathrm{i}}, \mathrm{v}_{\mathrm{i}} \\
& \ell\left(\mathrm{P}_{1}\right)=\ell\left(\mathrm{P}_{2}\right)=2 \mathrm{~s}-\mathrm{i}+3 \\
& \mathrm{~d}_{2}\left(\mathrm{v}_{1}, \mathrm{v}_{\mathrm{i}}\right)=2 \mathrm{~s}-\mathrm{i}+3 \\
& \text { ومن الواضح أن }
\end{aligned}
$$

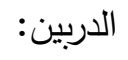

$$
\begin{aligned}
& \text { و وعليه فان } \\
& \text { عندما تكون } \\
& \text { من (6.2) و (6.3) نحصل على } \\
& \sum_{i=2}^{2 s+1} x^{d_{2}\left(v_{1}, v_{i}\right)}=\sum_{i=2}^{s+1} x^{i+1}+\sum_{i=s+2}^{2 s+1} x^{2 s-i+3}
\end{aligned}
$$

وبما أنه لكل قيمة من قيم í،

$\mathrm{d}_{2}\left(\mathrm{v}_{1}, \mathrm{v}_{\mathrm{i}}\right)=\mathrm{d}_{2}\left(\mathrm{v}_{1}, \mathrm{v}_{\mathrm{t}-\mathrm{i}+2}\right)$

فان هذه العلاقة تعطينا، وبعد التعويض عن قيمiا، المسافات للرأس vis نسبة إلى الرؤوس للجهة

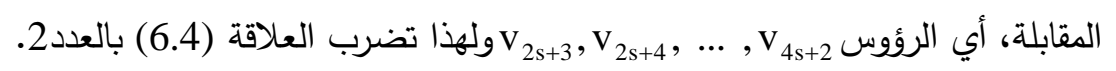

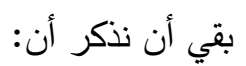

$\mathrm{d}_{2}\left(\mathrm{v}_{1}, \mathrm{v}_{2 s+2}\right)=3$

وبالاعتماد على ما تقدم نحصل على متعددة الحدود للمسافة العرضية-2 للرأس V ، وهي: $\mathrm{W}_{2}\left(\mathrm{v}_{1}, \mathrm{R}_{\mathrm{t}} ; \mathrm{x}\right)=\mathrm{x}^{3}+2 \sum_{\mathrm{i}=2}^{\mathrm{s+1}} \mathrm{x}^{\mathrm{i}+1}+2 \sum_{\mathrm{i}=\mathrm{s}+2}^{2 \mathrm{~s}+1} \mathrm{x}^{2 s-\mathrm{i}+3}$

ولما كان R منتظما بالنسبة إلى المسافة العرضية فان:

$$
\begin{aligned}
& \mathrm{W}_{\mathrm{w}}\left(\mathrm{R}_{\mathrm{t}} ; \mathrm{x}\right)=(\mathrm{t} / 2) \mathrm{W}_{\mathrm{w}}\left(\mathrm{v}_{1}, \mathrm{R}_{\mathrm{t}} ; \mathrm{x}\right) \\
& \text { حيث } 2 \text { س } 2 \text {. } \\
& \text { وأخيرا بالتعويض والتبسيط نحصل من (6.6) و (6.7) على (6.1) .وبهذا يتم البرهان. } \\
& \text { عبارة :6.3 إذا كانت t=4s، حيث } 2 \text { s ، فان: }
\end{aligned}
$$


$\mathrm{W}_{2}\left(\mathrm{R}_{\mathrm{t}} ; \mathrm{x}\right)=2 \mathrm{~s} \mathrm{x}^{3}+\mathrm{tx} \sum_{\mathrm{i}=2}^{\mathrm{s}} \mathrm{x}^{\mathrm{i}}+\mathrm{t} \mathrm{x}^{2} \sum_{\mathrm{i}=\mathrm{s}+1}^{2 \mathrm{~s}} \mathrm{x}^{2 \mathrm{~s}-\mathrm{i}}$

البرهان:

البيان R في الثكل 6.2 يمثل هذه الحالة.

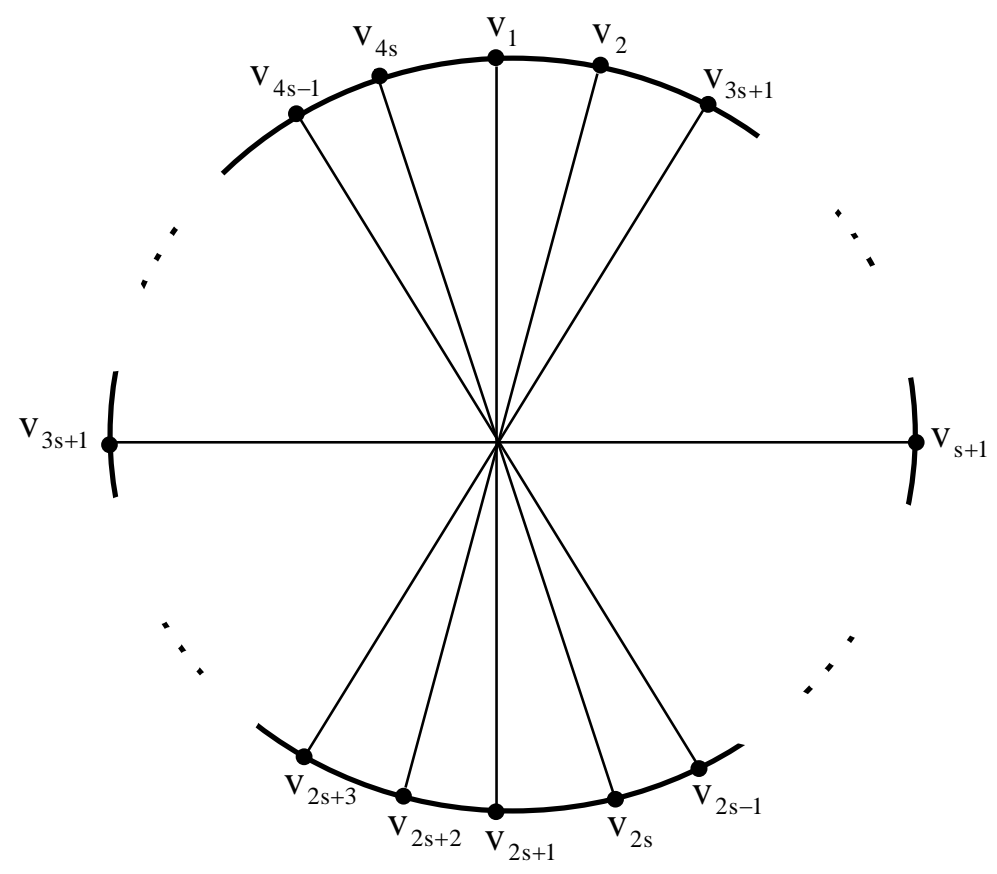

R الشكل6.2 بيان واكنر

كما في إثبات العبارة 6.2 فسوف نجد متعددة حدود وينر للمسافة العرضية-2 للرأس بسبب انتظام البيان بالنسبة إلى المسافة العرضية-W.

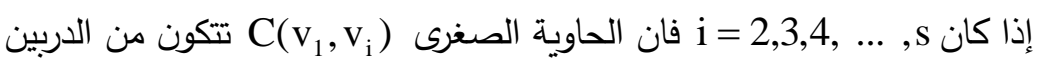

$$
\begin{gathered}
\mathrm{P}_{1}: \mathrm{v}_{1}, \mathrm{v}_{2}, \mathrm{v}_{3}, \ldots, \mathrm{v}_{\mathrm{i}} \\
\mathrm{P}_{2}: \mathrm{v}_{1}, \mathrm{v}_{2 \mathrm{~s}+1}, \mathrm{v}_{2 \mathrm{~s}+2}, \ldots, \mathrm{v}_{2 \mathrm{~s}+\mathrm{i}}, \mathrm{v}_{\mathrm{i}}
\end{gathered}
$$

$$
\text { ومن الواضح أن }
$$

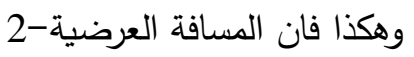

$\mathrm{d}_{2}\left(\mathrm{v}_{1}, \mathrm{v}_{\mathrm{i}}\right)=\mathrm{i}+1, \quad \mathrm{i}=2,3,4, \ldots, \mathrm{s}$ 


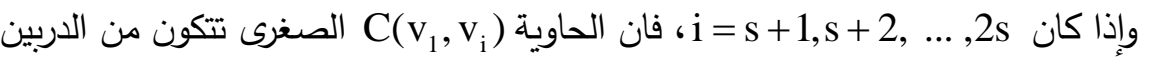

$$
\mathrm{P}_{1}: \mathrm{v}_{1}, \mathrm{v}_{2 \mathrm{~s}+1}, \mathrm{v}_{2 \mathrm{~s}}, \ldots, \mathrm{v}_{\mathrm{i}+2}, \mathrm{v}_{\mathrm{i}+1}, \mathrm{v}_{\mathrm{i}}
$$$$
\mathrm{P}_{2}: \mathrm{v}_{1}, \mathrm{v}_{4 \mathrm{~s}}, \mathrm{v}_{4 \mathrm{~s}-1}, \ldots, \mathrm{v}_{\mathrm{i}+2 \mathrm{~s}+2}, \mathrm{v}_{\mathrm{i}+2 \mathrm{~s}+1}, \mathrm{v}_{\mathrm{i}+2 \mathrm{~s}}, \mathrm{v}_{\mathrm{i}}
$$

$$
\ell\left(\mathrm{P}_{1}\right)=\ell\left(\mathrm{P}_{2}\right)=2 \mathrm{~s}-\mathrm{i}+2
$$

وواضح أن

$$
\text { وبهذا نحصل على المسافة العرضية-2 }
$$

$$
\mathrm{d}_{2}\left(\mathrm{v}_{1}, \mathrm{v}_{\mathrm{i}}\right)=2 \mathrm{~s}-\mathrm{i}+2, \quad \mathrm{i}=\mathrm{s}+1, \mathrm{~s}+2 \ldots, 2 \mathrm{~s}
$$

$$
\sum_{i=2}^{2 s} x^{d_{2}\left(v_{1}, v_{i}\right)}=\sum_{i=2}^{s} x^{i+1}+\sum_{i=s+1}^{2 s} x^{2 s-i+2}
$$

$$
\text { وهكذا من (6.9) و(6.10) نحصل على }
$$

$\mathrm{d}_{2}\left(\mathrm{v}_{1}, \mathrm{v}_{\mathrm{i}}\right)=\mathrm{d}_{2}\left(\mathrm{v}_{1}, \mathrm{v}_{\mathrm{t}-\mathrm{i}+2}\right)$

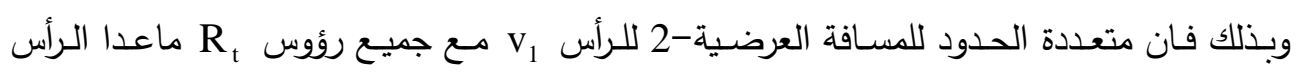
فان d $\mathrm{d}_{2}\left(\mathrm{v}_{1}, \mathrm{v}_{2 \mathrm{~s}+1}\right)=3$ هي ضعف متعددة الحدود $\mathrm{V}_{2 \mathrm{~s}+1}$ $\mathrm{W}_{2}\left(\mathrm{v}_{1}, \mathrm{R}_{\mathrm{t}} ; \mathrm{x}\right)=\mathrm{x}^{3}+2 \sum_{\mathrm{i}=2}^{\mathrm{s}} \mathrm{x}^{\mathrm{i}+1}+2 \sum_{\mathrm{i}=\mathrm{s}+1}^{2 \mathrm{~s}} \mathrm{x}^{2 \mathrm{~s}-\mathrm{i}+2}$

$$
\text { وباستخدام (6.7) نحصل على (6.8) وبهذا يتم البرهان. }
$$

يمكن أن نوحد العبارتين6.2 و 6.3 في مبرهنة واحدة لسهولة الرجوع إليها. مبرهنة6.4: متعددة حدود وينر للمسافة العرضية-2 لبيان واكنر R برتبة t هي:

$$
\mathrm{W}_{2}\left(\mathrm{R}_{\mathrm{t}} ; \mathrm{x}\right)=\left\{\begin{array}{l}
2 \mathrm{~s} \mathrm{x}^{3}+\mathrm{tx} \sum_{\mathrm{i}=2}^{\mathrm{s}} \mathrm{x}^{\mathrm{i}}+\mathrm{t} \mathrm{x}^{2} \sum_{\mathrm{i}=\mathrm{s}+1}^{2 \mathrm{~s}} \mathrm{x}^{2 \mathrm{~s}-\mathrm{i}}, \quad \text { when } \mathrm{t}=4 \mathrm{~s}, \mathrm{~s} \geq 2 \\
(2 \mathrm{~s}+1) \mathrm{x}^{3}+\mathrm{t} \mathrm{x} \sum_{\mathrm{i}=2}^{\mathrm{s}+1} \mathrm{x}^{\mathrm{i}}+\mathrm{t} \mathrm{x}^{2} \sum_{\mathrm{i}=\mathrm{s}+2}^{2 s+1} \mathrm{x}^{2 \mathrm{~s}+1-\mathrm{i}}, \text { when } \mathrm{t}=4 \mathrm{~s}+2, \mathrm{~s} \geq 1
\end{array}\right.
$$

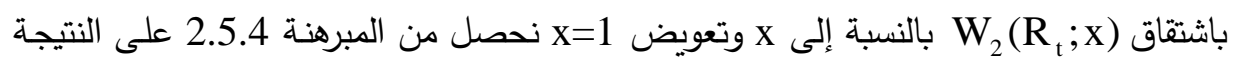


$\mathrm{W}_{2}\left(\mathrm{R}_{\mathrm{t}}\right)= \begin{cases}4 \mathrm{~s}\left(\mathrm{~s}^{2}+3 \mathrm{~s}-1 / 2\right), & \text { when } \mathrm{t}=4 \mathrm{~s}, \mathrm{~s} \geq 2 \\ 2 \mathrm{~s}\left(2 \mathrm{~s}^{2}+9 \mathrm{~s}+7\right)+3, & \text { when } \mathrm{t}=4 \mathrm{~s}+2, \mathrm{~s} \geq 1\end{cases}$

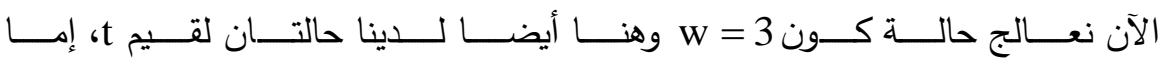

.) $s \geq 2($ ، $\mathrm{t}=4 \mathrm{~s} \quad \mathrm{~s} \geq 1)$ ، $t=4 \mathrm{~s}+2$

عبارة6.6: إذا كانت s

$\mathrm{W}_{3}\left(\mathrm{R}_{\mathrm{t}} ; \mathrm{x}\right)=(2 \mathrm{~s}+1) \mathrm{x}^{3}+\mathrm{t} \mathrm{x}^{3} \sum_{\mathrm{i}=2}^{\mathrm{s}+1} \mathrm{x}^{2 \mathrm{~s}-\mathrm{i}}+\mathrm{t} \sum_{\mathrm{i}=\mathrm{s}+2}^{2 \mathrm{~s}+1} \mathrm{x}^{\mathrm{i}-1}$

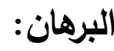

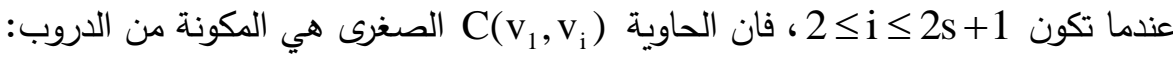

$\mathrm{P}_{1}: \mathrm{v}_{1}, \mathrm{v}_{2}, \mathrm{v}_{3}, \ldots, \mathrm{v}_{\mathrm{i}}$

$\mathrm{P}_{2}: \mathrm{v}_{1}, \mathrm{v}_{2 \mathrm{~s}+2}, \mathrm{v}_{2 \mathrm{~s}+1}, \ldots, \mathrm{v}_{\mathrm{i}+2}, \mathrm{v}_{\mathrm{i}+1}, \mathrm{v}_{\mathrm{i}}$

$\mathrm{P}_{3}: \mathrm{v}_{1}, \mathrm{v}_{4 \mathrm{~s}+2}, \mathrm{v}_{4 \mathrm{~s}+1}, \ldots, \mathrm{v}_{2 \mathrm{~s}+\mathrm{i}+1}, \mathrm{v}_{\mathrm{i}}$

$\ell\left(\mathrm{P}_{1}\right)=\mathrm{i}-1, \ell\left(\mathrm{P}_{2}\right)=\ell\left(\mathrm{P}_{3}\right)=2 \mathrm{~s}+3-\mathrm{i}$

ومن الواضح أن

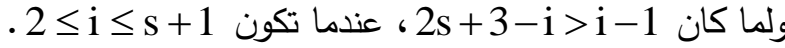

فانه لأجل 1 أن

$\mathrm{d}_{3}\left(\mathrm{v}_{1}, \mathrm{v}_{\mathrm{i}}\right)=2 \mathrm{~s}-\mathrm{i}+3$

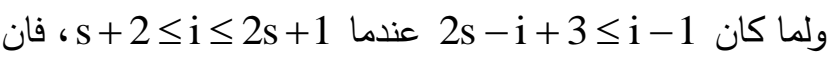

$\mathrm{d}_{3}\left(\mathrm{v}_{1}, \mathrm{v}_{\mathrm{i}}\right)=\mathrm{i}-1$

مهـا تقدم في (6.15) و(6.16) نجد أن متعـدة الحـدود للمسـافة العرضـية-3 بـين الـرأس

$\sum_{i=2}^{2 s+1} x^{d_{3}\left(v_{1}, v_{i}\right)}=\sum_{i=2}^{s+1} x^{2 s-i+3}+\sum_{i=s+2}^{2 s+1} x^{i-1}$ والرؤوس

$\mathrm{d}_{3}\left(\mathrm{v}_{1}, \mathrm{v}_{2 \mathrm{~s}+2}\right)=3$

ونلاحظ أن

$\mathrm{d}_{3}\left(\mathrm{v}_{1}, \mathrm{v}_{\mathrm{i}}\right)=\mathrm{d}_{3}\left(\mathrm{v}_{1}, \mathrm{v}_{\mathrm{t}-\mathrm{i}+2}\right)$

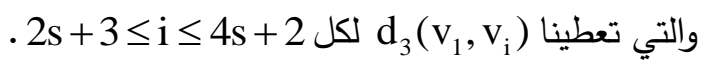


ولهذا تضرب العلاقة (6.17) بالعدد 2 وبعد جمعها مع X3 التي تتتج من (6.18) نحصل

$\mathrm{W}_{3}\left(\mathrm{v}_{1}, \mathrm{R}_{\mathrm{t}} ; \mathrm{x}\right)=\mathrm{x}^{3}+2 \sum_{\mathrm{i}=2}^{\mathrm{s}+1} \mathrm{x}^{2 s-\mathrm{i}+3}+2 \sum_{\mathrm{i}=\mathrm{s}+2}^{2 s+1} \mathrm{x}^{\mathrm{i}-1}$

وأخيرا، بالاستتاد إلى (6.7) وتبسيط الكقدار نحصل على (6.14) وبهذا يتم البرهان.

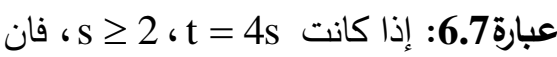

$\mathrm{W}_{3}\left(\mathrm{R}_{\mathrm{t}} ; \mathrm{x}\right)=2 \mathrm{~s} \mathrm{x}^{3}+\mathrm{tx} \mathrm{x}^{2} \sum_{\mathrm{i}=2}^{s+1} \mathrm{x}^{2 \mathrm{~s}-\mathrm{i}}+\mathrm{t} \sum_{\mathrm{i}=\mathrm{s}+2}^{2 \mathrm{~s}} \mathrm{x}^{\mathrm{i}-1}$

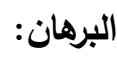

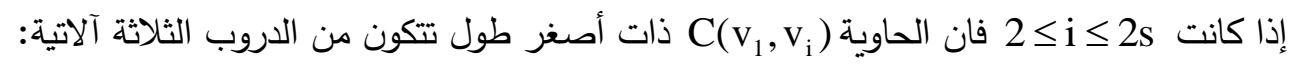
$\mathrm{P}_{1}: \mathrm{v}_{1}, \mathrm{v}_{2}, \mathrm{v}_{3}, \ldots, \mathrm{v}_{\mathrm{i}}$

$\mathrm{P}_{2}: \mathrm{v}_{1}, \mathrm{v}_{2 \mathrm{~s}+1}, \mathrm{v}_{2 \mathrm{~s}}, \mathrm{v}_{2 \mathrm{~s}-1}, \ldots, \mathrm{v}_{\mathrm{i}+1}, \mathrm{v}_{\mathrm{i}}$

$\mathrm{P}_{3}: \mathrm{v}_{1}, \mathrm{v}_{4 \mathrm{~s}}, \mathrm{v}_{4 \mathrm{~s}-1}, \mathrm{v}_{4 \mathrm{~s}-2}, \ldots, \mathrm{v}_{\mathrm{i}+2 \mathrm{~s}+2}, \mathrm{v}_{\mathrm{i}+2 \mathrm{~s}+1}, \mathrm{v}_{\mathrm{i}+2 \mathrm{~s}}, \mathrm{v}_{\mathrm{i}}$

$\ell\left(\mathrm{P}_{1}\right)=\mathrm{i}-1, \ell\left(\mathrm{P}_{2}\right)=\ell\left(\mathrm{P}_{3}\right)=2 \mathrm{~s}-\mathrm{i}+2$

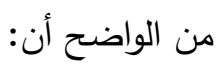

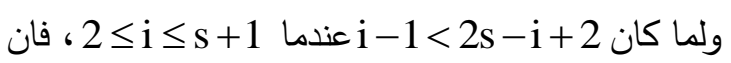

$\mathrm{d}_{3}\left(\mathrm{v}_{1}, \mathrm{v}_{\mathrm{i}}\right)=2 \mathrm{~s}-\mathrm{i}+2$

ولما كان 6.23)

$\mathrm{d}_{3}\left(\mathrm{v}_{1}, \mathrm{v}_{\mathrm{i}}\right)=\mathrm{i}-1, \quad \mathrm{~s}+2 \leq \mathrm{i} \leq 2 \mathrm{~s}$

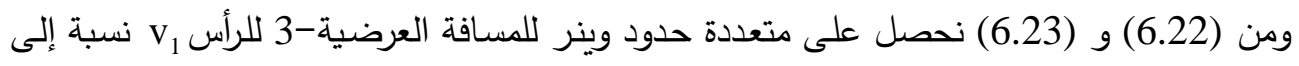

$\sum_{i=2}^{2 s} x^{d_{3}\left(v_{1}, v_{i}\right)}=\sum_{i=2}^{s+1} x^{2 s-i+2}+\sum_{i=s+2}^{2 s} x^{i-1}$

الرؤوس

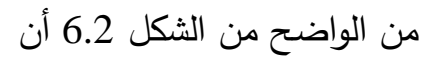

$\mathrm{d}_{3}\left(\mathrm{v}_{1}, \mathrm{v}_{2 s+1}\right)=3$

$\mathrm{d}_{3}\left(\mathrm{v}_{1}, \mathrm{v}_{\mathrm{i}}\right)=\mathrm{d}_{3}\left(\mathrm{v}_{1}, \mathrm{v}_{\mathrm{t}-\mathrm{i}+2}\right)$

وأخيرا نلاحظ أن لكل $2 \leq$ i 
وتكون

$$
\begin{aligned}
& \text { v وهذه تعطينا المسافة العرضية-3 للرأس v vنبة إلى الرؤوس }
\end{aligned}
$$

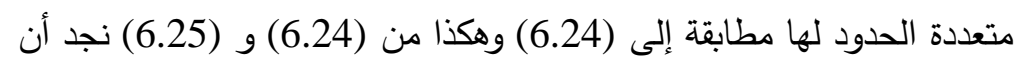

$$
\begin{aligned}
& \mathrm{W}_{3}\left(\mathrm{v}_{1}, \mathrm{R}_{\mathrm{t}} ; \mathrm{x}\right)=2 \sum_{\mathrm{i}=2}^{\mathrm{s}+1} \mathrm{x}^{2 \mathrm{~s}-\mathrm{i}+2}+2 \sum_{\mathrm{i}=\mathrm{s}+2}^{2 \mathrm{~s}} \mathrm{x}^{\mathrm{i}-1}+\mathrm{x}^{3} \\
& \text { وباستخدام (6.7) نحصل على (6.21) وبهذا يتم البرهان. }
\end{aligned}
$$

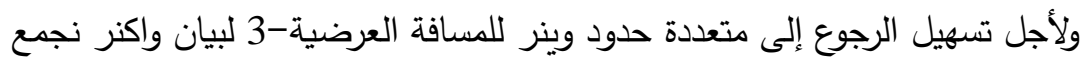

$$
\begin{aligned}
& \text { العبارتين6.6 و } 6.7 \text { في المبرهنة الآتية: } \\
& \text { مبرهنة6.8: متعددة حدود وينر للمسافة العرضية-3 للبيان Rي: } \\
& \mathrm{W}_{3}\left(\mathrm{R}_{\mathrm{t}} ; \mathrm{x}\right)=\left\{\begin{array}{l}
2 \mathrm{~s} \mathrm{x}^{3}+\mathrm{t} \mathrm{x}^{2} \sum_{\mathrm{i}=2}^{\mathrm{s}+1} \mathrm{x}^{2 \mathrm{~s}-\mathrm{i}}+\mathrm{t} \sum_{\mathrm{i}=\mathrm{s}+2}^{2 \mathrm{~s}} \mathrm{x}^{\mathrm{i}-1}, \text { when } \mathrm{t}=4 \mathrm{~s}, \mathrm{~s} \geq 2 \\
(2 \mathrm{~s}+1) \mathrm{x}^{3}+\mathrm{t} \mathrm{x}^{3} \sum_{\mathrm{i}=2}^{\mathrm{s}+1} \mathrm{x}^{2 \mathrm{~s}-\mathrm{i}}+\mathrm{t} \sum_{\mathrm{i}=\mathrm{s}+2}^{2 s+1} \mathrm{x}^{\mathrm{i}-1}, \text { when } \mathrm{t}=4 \mathrm{~s}+2, \mathrm{~s} \geq 1
\end{array}\right.
\end{aligned}
$$

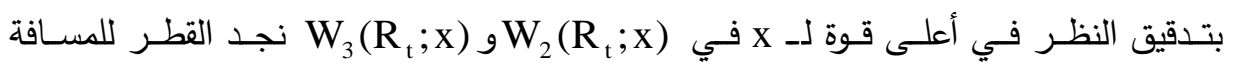
العرضية-2 و -3 للبيان Rو

$$
\begin{array}{r}
\operatorname{diam}_{2} \mathrm{R}_{\mathrm{t}}=1+\left\lceil\frac{\mathrm{t}}{4}\right\rceil \\
\operatorname{diam}_{3} \mathrm{R}_{\mathrm{t}}=\frac{1}{2} \mathrm{t}
\end{array}
$$

نتيجة6.10 : دليل وينر للمسافة العرضية-3 لبيان واكنر R هو:

$W_{3}\left(R_{t}\right)=\left\{\begin{array}{l}s(3 t s-t+6), \quad \text { when } t=4 s \\ s(3 t s+2 t+6)+3, \text { when } t=4 s+2\end{array}\right.$ 


\section{المصادر}

[1] A. S. Aziz (2007) "The Width Distance and the w-Wiener polynomials of a graph”, M. Sc. Thesis , Mosul University , Mosul.

[2] F. Buckley and F. Harary (1990) Distance in Graphs , AddisonWesley, Redwood.

[3] G. Chartrand and L. Lesniak (1986) Graphs and Digraphs, Wadsworth Inc. Belmont, California.

[4] R. Diestel (2000) Graph Theory , Springer-Verlag,New York.

[5] A. A. Dobrynin (1993) "On Decomposition of the Wiener Index for Graphs of Catacondensed Hexagonal System", Graph Theory Notes of New York, XXV , The New York Academy of Sciences , PP.19-22.

[6] I. Gutman (1993) "Some Properties of the Wiener Polynomial", Graph Theory Notes of New York, XXV, The New York Academy of Sciences , PP.13-18.

[7] H. Hosoya (1988) "On Some Counting Polynomials in Chemistry", Discrete Applied Math., 19 , PP.239-257.

[8] B. E. Sagan; Y-N. Yeh and P. Zhang (1996) "The Wiener Polynomial of a Graph", Intern. J. of Quantum Chemistry, Vol. 60, PP. 959-969. 